\title{
Assessment of Endothelial Function in Patients with Takayasu Arteritis by Measuring Flow-Mediated Vasodilation and Correlating it with Plasma Levels of Reactive Nitrogen Intermediates
}

\author{
Nagendra Boopathy Senguttuvan¹, Sanjay Jain², Ravindran Rajendran³, Veena Dhawan, Ajay Bahl5, Subhash C. Varma ${ }^{6}$ \\ ${ }^{1}$ Assistant Professor, Department of Cardiology, SRMC, Porur, Chennai, Tamilnadu, India. \\ ${ }^{2}$ Professor, Department of Internal Medicine, PGIMER, Chandigarh, Punjab, India. \\ ${ }^{3}$ Assistant Professor, Department of Cardiology, Trichy SRMMCH\&RC, Irungalur, Trichy, Tamilnadu, India. \\ 4Professor, Department of Pathology, PGIMER, Chandigarh, Punjab, India. \\ 5Professor, Department of Pathology, PGIMER, Chandigarh, Punjab, India. \\ ${ }^{6}$ Professor, Department of Internal Medicine, PGIMER, Chandigarh, Punjab, India.
}

\section{ABSTRACT}

\section{BACKGROUND}

Takayasu arteritis (TA) is the most common cause of renovascular hypertension in young Asians. We wanted to assess endothelial function in patients with TA using flow-mediated vasodilation (FMD) and its correlation with plasma levels of reactive nitrogen intermediates (RNI).

\section{METHODS}

Twenty patients with TA and 20 age- and sex-matched controls were studied. After obtaining informed consent, a detailed clinical examination and appropriate laboratory investigations were performed to assess disease activity (active and inactive groups as per American College of Rheumatologists criteria), FMD (measured as per the guidelines) and levels of RNI were measured.

\section{RESULTS}

The mean RNI level of patients with TA was $22.54 \mu \mathrm{M}$, which was significantly higher than that of controls $(5.53 \mu \mathrm{M})(\mathrm{p}<0.0001)$. The mean RNI level in patients with active disease (16 of 20 patients) was $26.97 \pm 17.43 \mu \mathrm{M}$, which was significantly higher than their counterparts in remission $(12.21 \pm 11.82 \mu \mathrm{M})$. However, the post hoc analysis of RNI levels did not demonstrate any statistically significant difference between patients with inactive disease and controls $(p=0.36)$. The mean FMD in patients with TA was found to be $25.72 \%$ compared with $24.83 \%$ in controls ( $p>0.05$ ).

\section{CONCLUSIONS}

Endothelial function, as measured by FMD, showed no difference between patients with TA and controls; however, mean RNI level was significantly elevated in patients with TA. Interestingly, patients with active TA had significantly higher RNI levels than those with inactive disease.

\section{KEY WORDS}

Endothelial Function, Flow-Mediated Vasodilation, Reactive Nitrogen Intermediates, Takayasu Arteritis
Corresponding Author: Dr. Ravindran Rajendran, Assistant Professor and HOD Department of Cardiology, Trichy, SRMMCH\&RC, Irungalur, Trichy-621105, Tamilnadu, India. E-mail: rravindranrajendran@gmail.com

DOI: $10.14260 / \mathrm{jemds} / 2019 / 558$

Financial or Other Competing Interests: None.

How to Cite This Article:

Senguttuvan NB, Jain S, Rajendran R, et al. Assessment of endothelial function in patients with takayasu arteritis by measuring flow-mediated vasodilation and correlating it with plasma levels of reactive nitrogen intermediates. J. Evolution Med. Dent. Sci. 2019;8(32):2563-2567, DOI: 10.14260/jemds/2019/558

Submission 03-07-2019, Peer Review 01-08-2019, Acceptance 03-08-2019, Published 12-08-2019. 


\section{BACKGROUND}

Takayasu arteritis (TA) is the most common cause of renovascular hypertension in India.(1) In the absence of a specific biomarker for this condition many clinical criteria have evolved to aid the diagnosis, of which the modified Sharma's criteria have been shown to have the highest sensitivity and specificity. In this involvement of subclavian artery forms a major criterion and if both are involved hypertension can remain masked when brachial artery pressure alone is monitored. Although it is classified as a large vessel vasculitis, it is the vasovasorum $(2,3)$ that is considered to be initially involved in the pathogenesis of TA. It is well known that diffuse endothelial dysfunction predisposes to an enhanced risk of cardiovascular morbidity and mortality.(4) Chronic high-grade inflammation in patients with TA is shown to promote endothelial dysfunction. ${ }^{(5)}$ Hence, the aim of our study was to study endothelial function in patients with TA by examining flow-mediated vasodilation (FMD) of the brachial artery, and measuring the levels of reactive nitrogen intermediates (RNI) and correlating them with disease activity.

\section{METHODS}

We screened 104 patients with TA ( $>18$ years of age, diagnosed by modified Sharma's criteria 1995(6) and enrolled 20 of them along with 20 age- and sex-matched controls. Patients with hypertension crisis, bilateral subclavian artery disease (41) and those who had undergone vascular surgeries/ angioplasties (33) were excluded from the study. With a prevalence of 2.5 cases of Takayasu arteritis per million populations, the above exclusion criteria and being a pilot to study, the above sample size was finalized. After institutional ethics committee clearance and informed consent from participants, both patients and controls underwent a detailed clinical examination and laboratory investigations that included complete hemogram with erythrocyte sedimentation rate, urea, creatinine, liver function tests, urine analysis, lipid profile and determination of C-reactive protein levels. Endothelial function in these patients was studied using FMD of the brachial artery and by measuring RNI levels in the blood.

\section{Measurement of RNI}

Five millilitres of venous blood were used for the measurement of RNI. The procedure that was used for the measurement of plasma nitrite was based on the method described by Green et al.(7)

\section{FMD}

The FMD was studied as recommended.(8) Under standard conditions, the brachial artery diameter of patients and controls was measured at the onset of R-waves in electrocardiography by B-mode ultrasonography. Flow through the artery was measured using pulsed- Doppler velocity signal from a mid-artery sample. The cuff of the sphygmomanometer was inflated above the systolic pressure for $5 \mathrm{~min}$. Mid artery Doppler signal for the measurement of flow was measured once the cuff had been released and after $15 \mathrm{~s}$ to assess hyperaemic activity. The diameter of the vessel was measured at the end of $1 \mathrm{~min}$. FMD was expressed as the change in percentage of the baseline diameter. In the present study, endothelial dysfunction was considered to be present if FMD in the brachial artery was $<10 \%$.

\section{Statistical analysis}

Data were explored for normality using histograms, measures of skewness and kurtosis, as well as Kolmogorov-Smirnov test for normality. Sample estimates for various quantitative variables were calculated using descriptive univariate statistics in both patients and controls. Ninety-five per cent CIs were also calculated for sample means. Considering the small sample size and non-normality of quantitative variables in the study, nonparametric statistics were mainly used. The MannWhitney U test was used to compare patients' and controls' clinical characteristics such as age, duration of illness, blood pressure changes and markers of endothelial dysfunction such as RNI levels and FMD. Fisher's exact test was used to compare qualitative variables such as sex, disease activity and steroid use. Spearman correlation analysis was performed to determine strength of association among endothelial dysfunction markers and clinical characteristics. Mean RNI levels were compared among three groups of study participants (Disease active versus inactive versus controls) using the Kruskal-Wallis test with subsequent post hoc pairwise analysis using the Mann-Whitney U test. All statistical tests were two-tailed; $\mathrm{p}<0.05$ was considered to be statistically significant.

\section{RESULTS}

The mean age of the patients with TA was 27.35 years $(95 \% \mathrm{CI}$ 23.31 to 31.3 years) compared with 26.85 years ( $95 \%$ CI 23.17 to 30.52 years) in the control group ( $p>0.05)$.

\begin{tabular}{|c|c|}
\hline Artery & N (\%) \\
\hline Ascending aorta & $2(10)$ \\
\hline Arch of aorta & $4(20)$ \\
\hline Subclavian artery (Right:left) & $8: 10(40: 50)$ \\
\hline Carotid artery (Right:left) & $2: 3(10: 15)$ \\
\hline Descending thoracic aorta & $6(30)$ \\
\hline Abdominal aorta & $10(50)$ \\
\hline Celiac artery & $6(30)$ \\
\hline Superior mesenteric artery & $8(40)$ \\
\hline Renal artery (Right:left) & $8: 10(40: 50)$ \\
\hline Inferior mesenteric artery & $0(0)$ \\
\hline Common iliac artery & $4(20)$ \\
\hline Internal iliac artery & $4(20)$ \\
\hline External iliac artery & $1(5)$ \\
\hline Aortic regurgitation & $1(5.55)$ \\
\hline Table 1. Angiographic Findings in Patients with Takayasu Arteritis \\
\hline
\end{tabular}

\begin{tabular}{|c|c|c|c|}
\hline Variable & Patient & Control & $\mathbf{P}^{*}$ \\
\hline RNI, $\mu \mathrm{M}$ & $22.54(14.53-30.55)$ & $5.53(2.99-8.07)$ & $<0.0001$ \\
\hline Baseline diameter, mm & $35.65(31.74-39.55)$ & $29.10(27.20-31.01)$ & 0.001 \\
\hline $1 \mathrm{~min}$ post diameter, $\mathrm{mm}$ & $44.40(39.60-49.20)$ & $36.24(34.06-38.42)$ & 0.003 \\
\hline FMD, \% & $25.72(17.55-33.90)$ & 24.83(21.94-27.73) & 0.42 \\
\hline \multicolumn{4}{|c|}{$\begin{array}{c}\text { Table 2. Comparative Analysis of Reactive Nitrogen Intermediate } \\
\text { (RNI) Levels and Flow-Mediated Vasodilation (FMD) } \\
\text { in Patients and Controls }\end{array}$} \\
\hline
\end{tabular}




\begin{tabular}{|c|c|c|c|c|}
\hline Variable & $\begin{array}{c}\text { Disease Active } \\
\text { (n=14) }\end{array}$ & $\begin{array}{c}\text { Disease } \\
\text { Inactive (n=6) }\end{array}$ & $\begin{array}{c}\text { Control } \\
\text { (n=20) }\end{array}$ & p \\
\hline RNI Levels $\mu \mathrm{M}$ & $\begin{array}{c}26.97 \pm 17.43 \\
(16.91 \text { to } 37.04)\end{array}$ & $\begin{array}{c}12.21 \pm 11.82 \\
(-0.20 \text { to } 24.61)\end{array}$ & $\begin{array}{c}5.53 \pm 5.42 \\
(2.99 \text { to } 8.07)\end{array}$ & $<0.0001$ \\
\hline & $\begin{array}{c}27.66 \\
(17.69 \text { to } 37.62)\end{array}$ & $\begin{array}{c}21.20 \\
(1.58 \text { to } 40.81)\end{array}$ & $\begin{array}{c}24.83 \\
(21.94 \text { to } 27.73)\end{array}$ & 0.05 \\
\hline Table 3. Comparison of Reactive Nitrogen Intermediate (RNI) Levels \\
Among Patients with Active Takayasu Arteritis, \\
Inactive Takayasu Arteritis and Controls \\
\hline
\end{tabular}

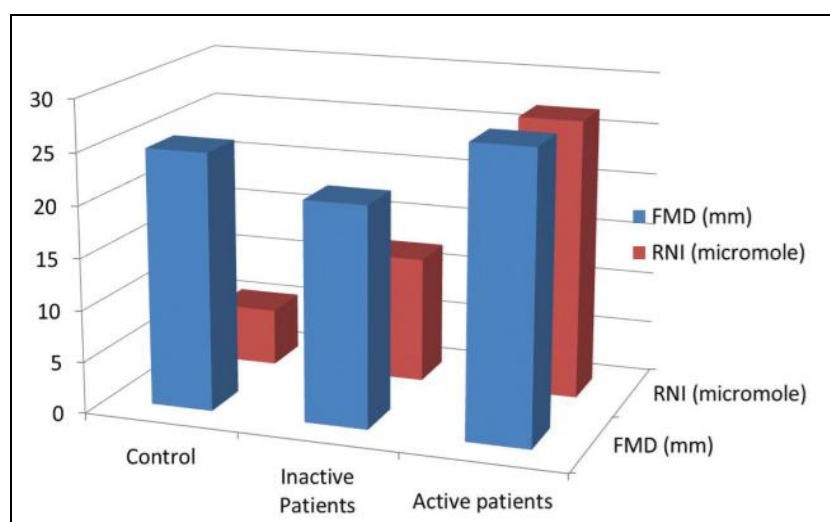

Figure 1. Comparison of Reactive Nitrogen Intermediate (RNI) Levels in Patients with Takayasu Arteritis According to Age and Disease Activity. FMD Flow-Mediated Vasodilation

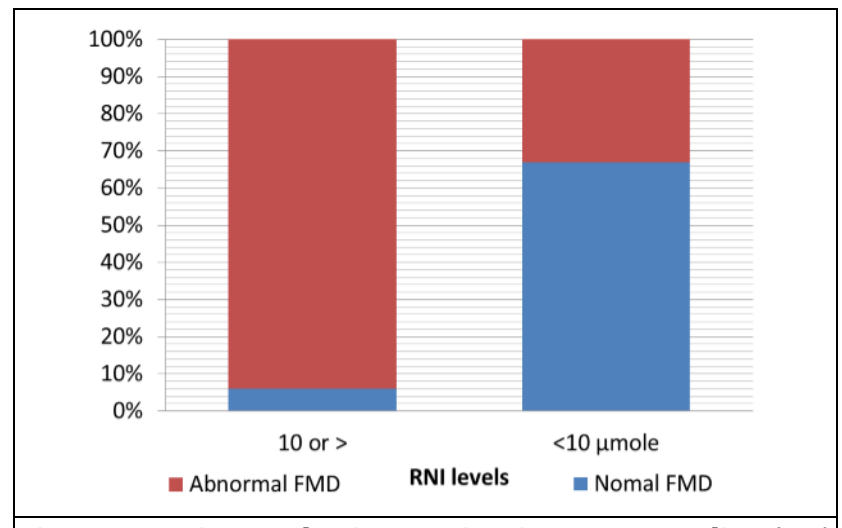

Figure 2. Bar Diagram Showing Reactive Nitrogen Intermediate (RNI) Levels and Flow-Mediated Vasodilation (FMD) (\%) in all Participants

\section{Angiographic Classification of Patients with TA}

The pattern of vascular involvement in TA patients is shown in Table 1 . The subclavian artery, renal artery and the abdominal aorta were the most commonly affected vessels in patients with TA. While there was no involvement of the inferior mesenteric artery, two patients with TA showed involvement of axillary artery and three patients had involvement of the brachial artery. The anterior tibial artery and femoral artery were involved in one patient each. Echocardiography was performed in 18 patients. One patient was found to have moderate aortic regurgitation (5.55\%) while myocardial involvement (Defined by diffuse hypokinesia or left ventricular ejection fraction $<50 \%$ ) was found in three patients $(16.65 \%)$.

\section{Disease Activity in TA Patients}

TA patients were classified into active and remission group based on the National Institutes of Health (USA) criteria (9). Sixteen $(70 \%)$ patients were found to have active disease while four $(30 \%)$ were found to be in remission. Twelve (92.9\%) patients with active TA had elevated inflammatory markers such as erythrocyte sedimentation rate and Creactive protein positivity $(\mathrm{p}<0.05)$. Systemic symptoms in the form of fever, arthralgia or myalgia were found in $71.4 \%$ of patients with active TA $(\mathrm{p}<0.05)$. Although carotidynia, another commonly used clinical marker for the disease activity, did not show a significant association with the disease activity, it has high positive predictive value for activity.

\section{Comparative Analysis of RNI in Patients and Controls}

The mean level of RNI in patients was $22.54 \mu \mathrm{M}(95 \%$ CI 14.53 $\mu \mathrm{M}$ to $30.55 \mu \mathrm{M})$ while it was $5.53 \mu \mathrm{M}(95 \%$ CI $2.99 \mu \mathrm{M}$ to 8.07 $\mu \mathrm{M}$ ) in controls, demonstrating that RNI levels were higher in TA patients $(\mathrm{p}<0.0001)$ (Table 2$)$.

\section{Comparative Analysis of FMD in Patients and Controls}

The mean baseline diameter was $35.65 \mathrm{~mm}$ (95\% CI $31.74 \mathrm{~mm}$ to $39.55 \mathrm{~mm}$ ) in patients and $29.10 \mathrm{~mm}$ (95\% CI $31.74 \mathrm{~mm}$ to $39.55 \mathrm{~mm}$ ) in controls $(\mathrm{p}<0.05)$. The mean $(95 \% \mathrm{CI})$ diameter of the brachial artery $1 \mathrm{~min}$ after the procedure was $44.40 \mathrm{~mm}$ ( $95 \%$ CI $39.60 \mathrm{~mm}$ to $49.20 \mathrm{~mm}$ ) in patients and $36.24 \mathrm{~mm}$ ( $95 \%$ CI $34.06 \mathrm{~mm}$ to $38.42 \mathrm{~mm}$ ) in controls $(\mathrm{p}<0.05)$. The mean FMD in patients was found to be $25.72 \%$ (95\% CI $17.55 \%$ to $33.89 \%$ ) compared with $24.83 \%$ (95\% CI $21.94 \%$ to $27.73 \%$ ) in controls ( $\mathrm{p}>0.05$ ) (Table 2).

\section{Disease Activity and Endothelial Function Markers}

The distribution of RNI levels were compared among patients stratified according to disease activity status (active versus inactive) and controls using Kruskal-Wallis test, which revealed highly significant differences among the aforementioned groups of participants $(\mathrm{p}<0.0001)$ (Table 3 ). Subsequent post hoc analysis was performed for pair-wise comparisons using a nonparametric test (Mann-Whitney U test), which showed that the mean RNI level was significantly higher in patients with active disease compared with controls $(\mathrm{p}<0.0001)$ as well as in patients with inactive disease $(\mathrm{p}=$ 0.04). This trend is further highlighted in Figure 1. However, post hoc analysis did not demonstrate any statistically significant difference between patients with inactive disease versus controls despite numerically higher mean RNI levels in former $(p=0.36)$. It was also found that there was no difference in FMD between active and inactive TA ( $p>0.05)$ (Table 3).

\section{RNI and FMD in TA}

TA patients and controls were subdivided based on their RNI levels, using an RNI level of $10 \mu \mathrm{M}$ as the cut-off value because RNI level $>10 \mu \mathrm{M}$ represented the 90th percentile in the control group, with only three subjects having an RNI level $>10$ $\mu \mathrm{M}$. The effect of higher of RNI levels in patients with TA was analysed in relation to FMD (\%) and found that patients with elevated RNI levels were found to have normal FMD $(p<0.05)$ (Figure 2). Also compared was the effect of having abnormal and normal FMD with abnormal and normal levels of RNI. All subjects belonging to the abnormal category of FMD belonged to the TA group regardless of their RNI levels. Most subjects with normal FMD belonged to the TA group if the RNI level is abnormal (ie, $>10 \mu \mathrm{M}$ ). 


\section{DISCUSSION}

We assessed the biology of nitric oxide (NO) in TA patients to determine its role in the disease activity of TA and endothelial function, and found that patients with TA had significantly higher RNI levels than controls. Vascular endothelium was considered to be a simple semipermeable membrane separating two compartments (i.e. intravascular and extravascular space). Presently, the endothelium is considered to be the largest organ in the body having sophisticated endocrine, paracrine and autocrine functions. It regulates various biological processes including fibrinolysis, haemostasis, lipid metabolism, blood pressure control, etc. Various factors have been shown to alter the function of endothelium leading to endothelial dysfunction. $\mathrm{NO}^{(10)}$ is a membrane-permeable free radical molecule synthesized by NO synthase (NOS) using arginine and oxygen as substrates. Three isoforms of NOS(11) were described and they are transcribed from three different genes in humans: nNOS (NOSI), inducible NOS (iNOS) (NOS 2) and endothelial (eNOS) (NOS3). The second isoform is inducible, while the rest are constitutively expressed. Normally, iNOS expression is high in myocytes, skeletal muscle and Purkinje cells. iNOS is also expressed during pathological states in human endothelial cells, synovial fibroblasts, polymorph nuclear cells, lymphocytes and natural killer cells.(11) iNOS produces several log-fold higher amounts of NO than the other isoforms. Lipopolysaccharide, interleukin (IL)-6, interferon- $\gamma$ (IFN $\gamma$ ), IL1 $\beta$, and tumour necrosis factor- $\alpha$ (TNF- $\alpha$ ) increase the expression of iNOS while TGF- $\beta$, IL-10 and IL-14 are found to decrease the expression of iNOS.(11) It is the balance between these cytokines that govern the activity of iNOS. iNOS can result in beneficial or detrimental outcome for an organism. NO produced acutely, as directed against a pathogen in an acute infection, is beneficial, while NO produced over a long period of time, as in some diseases such as systemic lupus erythematosus,(12) may be detrimental. The redox state inside the cell determines whether NO is used for the production of beneficial or harmful substances. When formed in the presence of $\mathrm{O}_{2}, \mathrm{NO}$ can oxidize to $\mathrm{NO}_{2}$ and $\mathrm{NO}_{3}$. In the presence of superoxide ( $\mathrm{SO}$ or $\mathrm{O}_{2 .-}-$ ). $\mathrm{NO}$ oxidizes to form peroxynitrite (ONOO-). Markers of systemic RNI production are also found to be increased in other autoimmune disease such as systemic lupus erythematosus with activity.(13) Similarly, we found that mean levels of RNI in patients with TA was significantly higher than in controls $(\mathrm{p}<0.0001)$. As discussed, both eNOS and iNOS could have contributed the elevated RNI levels in patients with TA. Increased NO due to diet was prevented in our patients because RNI samples were drawn after an adequate period of fasting. Fluid shear stress (the frictional force resulting from blood flow) is a principal regulator of eNOS expression.(14) TA patients have increased shear stress produced at the level of involved portion of the aorta and its branches, which can elevate eNOS messenger RNA expression. In contrast to steady laminar shear stress, steady hydrostatic pressure (hypertension) was shown to decrease eNOS messenger RNA levels.(15) If the elevated RNI levels in TA patients were all because of increased eNOS activity, then TA patients who had inactive disease should have also shown significantly higher RNI levels. Although our inactive patients had higher mean RNI levels than controls, it was not found to be statistically significant $(p>0.05)$. Hence, the elevated RNI levels should have been predominantly contributed by the iNOS isoform. In contrast to RNI levels, there was no significant relationship between disease activity and FMD. NO expressed by iNOS leads to vasodilation,(16) but it is shown that iNOS expression can also cause vasoconstriction by abnormal calcium signalling, generation of peroxynitrite and non-vasoactive oxidative products with catecholamines. These results support the hypothesis that NO from iNOS inhibits contractile responses during inflammation.(16-18) Hence, what was expected was a decrease in the post procedure arterial diameter in patients with TA. Instead of getting a decrease in the post-procedure diameter of brachial artery in patients with TA, no difference in FMD was observed between patients and controls. We hypothesize that it could have been because of the decreased inhibitory effect of iNOS on eNOS(16,18) that is stimulated through factors such as shear stress. It is well known that the expression of eNOS in the endothelium is decreased by the negative effect of iNOS while it is increased by the positive influence of fluid shear stress. The inhibitory effect of iNOS possibly would have been blunted by the shear stress-induced increase in the expression of eNOS, which was further increased by the procedure in these patients. Although most studies showed decreased FMD in systemic vasculitis patients, one study showed that there was an enhanced FMD in patients with systemic vasculitis(19) where they had postulated that continued endothelial activation, even when the disease was clinically quiescent, could have led to this response. Unlike the results reported by Espinola-Zavaleta et al,(20) endothelial function as measured by FMD was found to be similar between patients with TA and normal controls ( $p>0.05)$. Hoffmann et al(21) showed that the efficacy of infliximab in TA patients acts by inhibiting the action of TNF- $\beta$, thereby decreasing iNOS activity. By inhibiting NOS, in addition to inhibition of iNOS, we decrease the activity of eNOS and nNOS, predisposing patients to develop hypertension and cognitive dysfunction, respectively. Therefore, a drug that acts super-selectively on iNOS will avoid these complications. Pharmacological inhibition of iNOS has been performed in murine models of lupus using a number of competitive inhibitors of the Larginine substrate. For an inhibitor to be highly selective, it should be 50 - to 100 -fold more selective for iNOS than eNOS and nNOS. Hence, novel agents such as iNOS inhibitors may be useful in the management of patients with TA in the future and RNI can be used to assess the activity in patients with TA.

\section{Limitations}

We included only 20 patients with TA, which could have easily skewed the results on either side. However, obtaining a larger sample size may be difficult in diseases such as TA, given its incidence of three to five per million. We did not perform special studies to quantify the expression of specific isoforms of NOS to quantify the amount of RNI produced from each of the three isoforms.

\section{CONCLUSIONS}

We found that patients with TA were found to have significantly higher RNI levels than controls. Patients with active TA were found to have higher RNI levels compared with inactive TA. Endothelial function measured using FMD in patients with TA was similar to that of controls. 


\section{REFERENCES}

[1] Chugh KS, Jain S, Sakhuja V, et al. Renovascular hypertension due to Takayasu's arteritis among Indian patients. Q J Med 1992;85(307-308):833-43.

[2] Noris M. Pathogenesis of Takayasu's arteritis. J Nephrol 2001;14(6):506-13.

[3] Vaideeswar P, Deshpande JR. Pathology of Takayasu arteritis: a brief review. Ann Pediatr Cardiol 2013;6(1):52-8.

[4] Filer A, Nicholls D, Corston R, et al. Takayasu arteritis and atherosclerosis: illustrating the consequences of endothelial damage. J Rheumatol 2001;28(12):2752-3.

[5] Seyahi E, Ugurlu S, Cumali R, et al. Atherosclerosis in Takayasu arteritis. Ann Rheum Dis 2006;65(9):1202-7.

[6] Sharma BK, Jain S, Suri S, et al. Diagnostic criteria for Takayasu arteritis. Int J Cardiol 1996;Suppl 54:S141-7.

[7] Green LC, Wagner DA, Glogowski J, et al. Analysis of nitrate, nitrite and $[15 \mathrm{~N}]$ nitrate in biological fluids. Anal Biochem 1982;126(1):131-8.

[8] Corretti MC, Anderson TJ, Benjamin EJ, et al. Guidelines for the ultrasound assessment of endothelial-dependent flow-mediated vasodilation of the brachial artery: a report of the International Brachial Artery Reactivity Task Force. J Am Coll Cardiol 2002;39(2):257-65.

[9] Kerr GS, Hallahan CW, Giordano J, et al. Takayasu arteritis. Ann Intern Med 1994;120(11):919-29.

[10] Furchgott RF, Zawadzki JV. The obligatory role of endothelial cells in the relaxation of arterial smooth muscle by acetylcholine. Nature 1980;288:373-6.

[11] Nathan C. Inducible nitric oxide synthase: What difference does it make? J Clin Invest 1997;100(10):2417-23.

[12] Belmont HM, Levartovsky D, Goel A, et al. Increased nitric oxide production accompanied by the up-regulation of inducible nitric oxide synthase in vascular endothelium from patients with systemic lupus erythematosus. Arthritis Rheum 1997;40(10):1810-6.
[13] Wanchu A, Khullar M, Deodhar SD, et al. Nitric oxide synthesis is increased in patients with systemic lupus erythematosus. Rheumatol Int 1998;18(2):41-3.

[14] Malek AM, Izumo S, Alper SL. Modulation by pathophysiological stimuli of the shear stress-induced upregulation of endothelial nitric oxide synthase expression in endothelial cells. Neurosurgery 1999;45(2):334-44: discussion 344-5.

[15] Kleinert H, Pautz A, Linker K, et al. Regulation of the expression of inducible nitric oxide synthase. Eur J Pharmacol 2004;500(1-3):255-66.

[16] Gunnett CA, Lund DD, McDowell AK, et al. Mechanisms of inducible nitric oxide synthase-mediated vascular dysfunction. Arterioscler Thromb Vasc Biol 2005;25(8):1617-22.

[17] Zhang H, Du Y, Cohen RA, et al. Adventitia as a source of inducible nitric oxide synthase in the rat aorta. Am J Hypertens 1999;12(5):467-75.

[18] Ooboshi H, Chu Y, Rios CD, et al. Altered vascular function after adenovirus-mediated overexpression of endothelial nitric oxide synthase. Am J Physiol 1997;273(1 Pt 2):H265-70.

[19] Bruce IN, Harris CM, Nugent A, et al. Enhanced endothelium-dependent vasodilator responses in patients with systemic vasculitis. Scand J Rheumatol 1997;26(4):318-24.

[20] Espinola-Zavaleta N, Soto-López ME, Carreón-Torres E, et al. Altered flow-mediated vasodilatation, low paraoxonase-1 activity and abnormal high-density lipoprotein subclass distribution in Takayasu's arteritis. Circ J 2009;73(4):760-6.

[21] Hoffman GS, Merkel PA, Brasington RD, et al. Anti-tumor necrosis factor therapy in patients with difficult to treat Takayasu arteritis. Arthr Rheum 2004;50(7):2296-304. 